\title{
Granzyme B produced by Plasmacytoid Dendritic Cells Promotes Antigen uptake While Suppressing Premature T cell Activation
}

\author{
Dorit Fabricius $^{2 *}$, Timo Trzaska ${ }^{1}$ and Bernd Jahrsdörfer ${ }^{1 *}$ \\ ${ }^{1}$ Laboratory of Cellular Immunology Institute of Clinical Transfusion Medicine and Immunogenetics,Ulm University, \\ Helmholtzstrasse 10, 89081 Ulm, Germany \\ ${ }^{2}$ Department of Pediatrics,Ulm University,Eythstrasse 24,89075 Ulm, Germany
}

\begin{abstract}
Received: July 22, 2016; Accepted: August 12, 2016; Published: August 25, 2016
*Corresponding authors: Prof. Dr. Bernd Jahrsdörfer, M.D., Associate Professor of Clinical Pharmacology Laboratory of Cellular Immunology, Institute of Clinical Transfusion Medicine and Immunogenetics, Ulm University, Helmholtzstrasse 10, 89081 Ulm, Germany, Tel: +49 731150 6868; Fax: +49 731 150 575; E-mail: bernd.jahrsdoerfer@uni-ulm.de
\end{abstract}

PD Dr. med. Dorit Fabricius, M.D., Assistant Professor of Pediatrics, Department of Pediatrics, Ulm University, Eythstrasse 24,89075 Ulm, Germany, Tel: +49731 500 57376; Fax: +49 731500 57042; E-mail: doritfabricius@uni-ulm.de

The serine protease granzyme B (GzmB) is classically known to be an apoptogenic effector molecule produced by cytotoxic cells including NK cells and CD8+ T lymphocytes [1]. However,a series of further cell types are able to express GzmB, most of them in the absence of perforin.Below them are different antigen-presenting cells (APC) including B cells $[2,3]$, monocyte derived dendritic cells $[4,5]$ and plasmacytoid dendritic cells $[6,7]$. Meanwhile we know that GzmB contributes to a variety of APC-related functions. GzmB may therefore represent a promising novel target for the improvement of vaccination approaches based on the use of pDC and other dendritic cell types expressing GzmB.

Research in recent years has uncovered a variety of noncytotoxic roles of GzmB [8, 9]. Of note, the cytotoxic function of $\mathrm{T}$ cells and NK cells results from GzmB being secreted in the presence of the pore-forming protein perforin (Pfn). This allows GzmB to reach the cytosol of target cells after exocytosis, where it then activates apoptosis-inducing enzymes such as caspases, DNAses and BID [1]. In contrast, certain immune cells including plasmacytoid dendritic cells (pDC) $[6,10]$ and B cells $[11,12]$ can produce GzmB only, but not Pfn. GzmB secreted by these APC types therefore primarily encounters substrates with an extracellular or membrane-close localization such as extracellular proteins or membrane-bound receptors [8]. As a result, these cells develop a strong immune regulatory potential $[6,11,12]$ based on GzmBdependent cleavage of the T cell receptor (TCR)- $\zeta$-chain $[9,10$, 13]. Consequently, loss of the TCR- $\zeta$-chain directly limits the proliferative capacity and survival of effector $\mathrm{T}$ cells.

GzmB produced by APC however may not only play an important role for their interaction with $\mathrm{T}$ cells, but also for antigen processing. Recently, GzmB was shown to enhance both the uptake of antigens by release of eat-me signals on dying cells as well as their cross-presentation $[14,15]$. Of note, we were able to confirm these data in an in-vitro model, which demonstrated that pDC are able to take up antigens derived from lysed tumor cells in a GzmB-dependent manner (Fig1).

In addition to the impact GzmB has on antigen uptake, the way it cleaves peptides can result in fragments that represent neo-antigens, allowing the establishment of increased immune responses towards such antigens $[16,17]$. Thus, one reason for

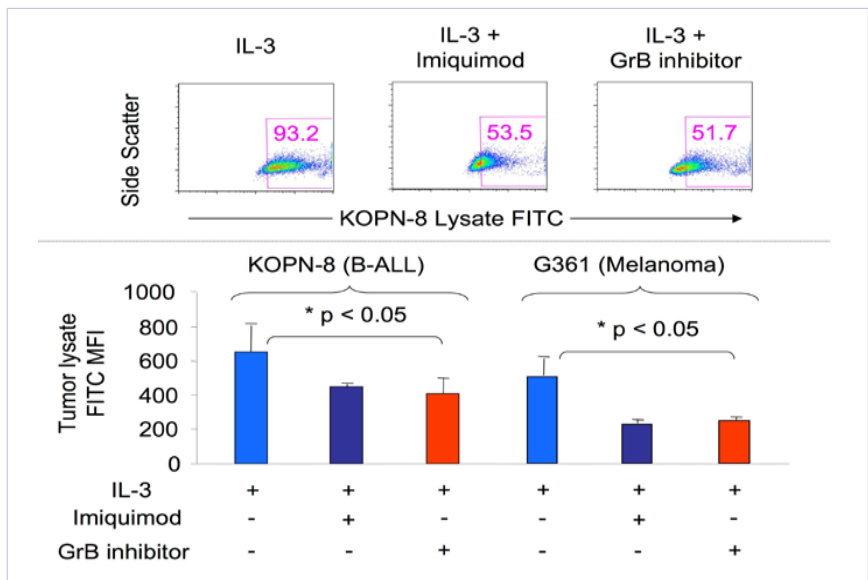

Figure 1: $5 \times 10^{7}$ tumor cells of the B cell precursor leukemia cell line KOPN-8 and the melanoma cell line G361 were irradiated with $60 \mathrm{~Gy}$ and lysed by 6 subsequent freeze-thaw cylces. Then, protein content was normalized and lysate was labeled using the Fluo Reporter FITC protein labeling kit by Molecular Probes. Purified pDC were plated in the presence of IL-3, imiquimod and a GzmB substrate inhibitor as indicated. Then, lysate was added for $24 \mathrm{hrs}$. Finally, pDC were harvested and analyzed by FACS. Dot plots show results from one representative experiment with KOPN-8 lysate out of three with similar results. Bar graphs represent average values from three independent experiments, error bars indicate SEM. 


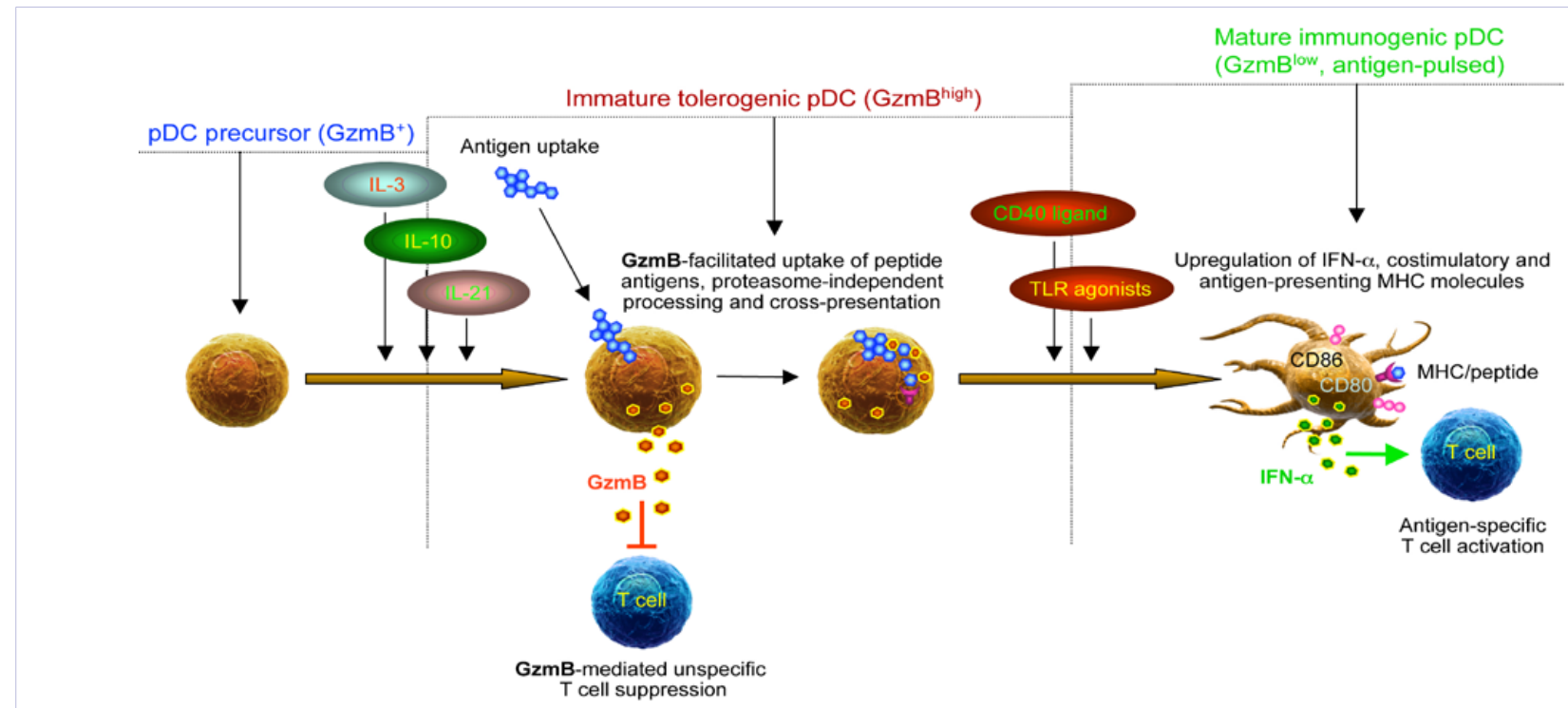

Figure 2: Maturation of pDC precursors occurs in a biphasic process. During the first phase, pDC develop a tolerogenic phenotype in response to different cytokines including IL-3,IL-10 and IL-21. This phenotype involves the production of large amounts of the serine protease GzmB. GzmB is not only able to suppress effector $\mathrm{T}$ cell responses, but is also involved in antigen uptake and proteasome-independent antigen processing. In the second phase, pDCs are rapidly (within 24 hours) matured in response to TLR ligands and CD40 ligand. GzmB is down modulated, while co-stimulatory molecules and $\mathrm{MHC}$ /peptide complexes are simultaneously up regulated, resulting in a highly immunogenic and mature APC phenotype.

the development of autoimmune diseases may be the preferential degradation of antigens by GzmB, for example in the course of $\mathrm{T}$ cell-mediated attack of virus-infected cells. This suggests that antigens degraded by GzmB may be more immunogenic than antigens produced by other proteases. The reason why certain APC including pDC express GzmB may therefore be manifold. On the one hand, pDC-secreted GzmB may halt down unspecific T cell activation, while antigen loading and processing is ongoing. At the same time, GzmB may facilitate antigen uptake and processing into immunogenic fragments for cross-presentation (Fig. 2). Overall, our data suggest that the dynamics of GzmB expression by pDC may strongly impact on their efficacy to induce antigen specific cellular immune responses. Further studies are necessary to elucidate, whether or not GzmB produced by APC may serve as a major target for the therapeutic manipulation of immune responses after vaccination.

\section{References}

1. Lord S J, RV Rajotte, GS Korbutt, RC Bleackley. Granzyme B: a natural born killer. Immunol Rev. 2003;193:31-38.

2. Jahrsdörfer B, SE Blackwell, JE Wooldridge, J Huang, MW Andreski, LS Jacobus, et al. B-chronic lymphocytic leukemia cellsand other B cells can produce granzyme $B$ and gain cytotoxic potential after interleukin21-based activation. Blood. 2006;108(8):2712-2719. D0I:10.1182/ blood-2006-03-014001.

3. Hagn M, E Schwesinger V, Ebel K, Sontheimer, J Maier, T Beyer, et al. Human B cells secrete granzyme B when recognizing viral antigens in the context of the acute phase cytokineIL-21. J Immunol. 2009;183(3):1838-1845. doi:10.4049/jimmunol.0901066.

4. Schuldhaus C, T Beyer, C Kaltenmeier, S Lindner, H Schrezenmeier, B Jahrsdörfer. Monocytes express the serine protease granzyme B - potential involvement in antigen processing and presentation. J Immunol. 2013;190 (1 Suppl):110-117.
5. Korthals M, N Safaian, R Kronenwett, D Maihofer, M Schott, C Papewalis, et al. Monocyte derived dendritic cells generated by IFN-alpha acquire mature dendritic and natural killer cell properties as shown by gene expression analysis. J Transl Med. 2007;5:46. DOI:10.1186/14795876-5-46.

6. Jahrsdörfer B, A Vollmer, SE Blackwell, J Maier, K Sontheimer, T Beyer, et al. Granzyme B produced by human plasmacytoid dendritic cells suppresses T-cell expansion. Blood. 2010;115(6):11561165. doi:10.1182/blood-2009-07-235382.

7. Rissoan MC, T Duhen, JM Bridon, N Bendriss-Vermare, C Peronne, B de Saint Vis, et al. Subtractive hybridization reveals the expression of immunoglobulin-like transcript 7, Eph-B1, granzyme B, and 3 novel transcripts in human plasmacytoid dendritic cells. Blood. 2002;100(9):3295-3303. DOI:10.1182/blood-2002-02-0638.

8. Buzza MS, PI Bird. Extracellular granzymes: current perspectives. Biol Chem. 2006;387(7):827-837. DOI:10.1515/BC.2006.106.

9. Hagn M, B Jahrsdörfer. Why do human B cells secrete granzyme B? Insights into a novel B-cell differentiation pathway. Oncoimmunology. 2012;1(8):1368-1375. doi:10.4161/onci.22354.

10. Fabricius D, B Nussbaum, D Busch, V Panitz, B Mandel, A Vollmer, et al. Antiviral vaccines license $\mathrm{T}$ cell responses by suppressing granzyme B levels in human plasmacytoid dendritic cells. J Immunol. 2013;191(3):1144-1153. doi:10.4049/jimmunol.1203479.

11.Lindner S, K Dahlke, K Sontheimer, M Hagn, C Kaltenmeier, TF Barth, et al. Interleukin 21-Induced Granzyme B-Expressing B Cells Infiltrate Tumors and Regulate T Cells. Cancer Res. 2013;73(8):2468-2479. doi:10.1158/0008-5472.CAN-12-3450.

12. Kaltenmeier C, A Gawanbacht, $\mathrm{T}$ Beyer, $\mathrm{S}$ Lindner, $\mathrm{T}$ Trzaska, JA van der Merwe, et al. CD4+ T cell-derived IL-21 and deprivation of CD40 signaling favor the in vivo development of granzyme B-expressing regulatory B cells in HIV patients. Journal of immunology. 2015;194(8):3768-3777. doi:10.4049/jimmunol.1402568. 
13. Wieckowski E, GQ Wang, BR Gastman, LA Goldstein, H Rabinowich. Granzyme B-mediated degradation of T-cell receptor zeta chain Cancer Res. 2002;62(17):4884-4889.

14. Hoves S, VR Sutton, NM Haynes, ED Hawkins, D Fernandez Ruiz $\mathrm{N}$ Baschuk, et al. A critical role for granzymes in antigen crosspresentation throughregulating phagocytosis of killed tumor cells. Immunol. 2011;187: 1166-1175. doi:10.4049/jimmunol.1001670.

15. Hoves S, VR Sutton, JA Trapani. A novel role for granzymes in antitumor immunity. Oncoimmunology. 2012;1(2):219-221. DOI:10.4161/ onci.1.2.18102
16. Casciola-Rosen L, F Andrade, D Ulanet, WB Wong, A Rosen. Cleavage by granzyme $B$ is strongly predictive of autoantigen status: implications for initiation of autoimmunity. The Journal of experimental medicine. 1999;190(6):815-826.

17. Casciola-Rosen L, A Miagkov, K Nagaraju, F Askin, L Jacobson, A Rosen, DB Drachman. Granzyme B: evidence for a role in the origin of myasthenia gravis. J Neuroimmunol. 2008;201-202:33-40. doi:10.1016/j.jneuroim.2008.04.041. 